\title{
Hybrid Silicon Lasers
}

\author{
Günther Roelkens ${ }^{1}$, Yannick De Koninck ${ }^{1}$, Shahram Keyvaninia ${ }^{1}$, Stevan Stankovic ${ }^{1}$, Martijn \\ Tassaert $^{1}$, Marco Lamponi ${ }^{2}$, Guanghua Duan ${ }^{2}$, Dries Van Thourhout ${ }^{1}$ and Roel Baets ${ }^{1}$ \\ ${ }^{1}$ Department of Information Technology, Photonics Research Group, Ghent University - IMEC \\ Sint-Pietersnieuwstraat 41 B-9000 Ghent, Belgium \\ 2 Alcatel-Thales III-V labs, Campus Polytechnique, 1, Avenue A. Fresnel, 91767 Palaiseau \\ cedex, France
}

\begin{abstract}
Hybrid silicon lasers based on bonded III-V layers on silicon are discussed with respect to the challenges and trade-offs in their design and fabrication. Focus is on specific designs that combine good light confinement in the gain layer with good spectral control provided by grating structures patterned in silicon.
\end{abstract}

\section{INTRODUCTION}

Silicon-based photonic integrated circuits are gaining considerable importance for a variety of applications, from telecommunications to sensors. The interest in this technology stems mostly from the expectation that the maturity and low cost of CMOS-technology can be applied for advanced photonics products. Other driving forces for silicon photonics include the design richness associated with high refractive index contrast as well as the potential for integration of photonics with electronics.

Building light sources, and in particular laser sources, on integrated silicon circuits is a long sought goal, on one hand in order to complete the functionality of the integrated circuit with one or several light sources but on the other hand also as a manufacturing approach for lasers on large wafers in CMOS-fabs. In terms of device performance the most successful approach to date is definitely the hybrid (also called heterogeneous) III-V on silicon laser. In this device thin layers of III-V semiconductors are bonded to silicon. The laser cavity gets its gain from the III-V layers but couples its output light into a silicon waveguide. Often part of the cavity structure is implemented by means of patterning in silicon, thereby taking advantage of the resolution and accuracy of lithography tools in CMOS fabs. In that sense these hybrid III-V/silicon lasers take the best of two worlds.

In spite of rapid progress in this field since about 2006 the design and fabrication of hybrid silicon lasers present specific challenges and trade-offs. There are many choices to be made, both in terms of the cavity structure, the optical coupling between the silicon waveguide and the III-V waveguide and the technological approach. A relatively large variety of approaches have been reported in the past years.

In this paper we will first review the options in the design of hybrid laser cavities, with emphasis on the light distribution in and the light exchange between the silicon and III-V layer. This will be followed by a section on the technological challenges presented by the bonding process. We will then focus on specific designs that combine good light confinement in the gain layer with good spectral control provided by grating structures patterned in silicon.

\section{HYBRID LASER CAVITY DESIGN}

Although the basic device cross-section, a III-V epitaxial layer transferred to a silicon-on-insulator waveguide wafer, is very simple, there is a large degree of freedom in the design of the laser cavity. Particularly, in the design a choice needs to be made whether the optical mode is predominantly confined in the silicon waveguide or in the III-V overlay. Both options have their advantages and disadvantages and they are not even mutually exclusive, given the fact that e.g. adiabatic tapers inside the laser cavity can be used to change the optical 
confinement in the respective layers. Confining the optical mode predominantly in the silicon waveguide layer has the advantage of making the coupling to a passive silicon waveguide straightforward. Moreover, wavelength selective features can easily be defined in the silicon waveguide layer using CMOS fabrication techniques, which provides an accurate mechanism to control the emission wavelength of the laser. A drawback of this siliconconfined approach however is that only a small fraction of the optical mode interacts with the gain material, resulting in longer laser cavities and higher power consumption devices. This type of laser cavity is illustrated in figure 1(a). ${ }^{1}$ On the other side of the design space, one can find III-V on silicon laser geometries, where the optical mode is completely confined in the III-V waveguide layer and where the optical cavity is defined in the III-V semiconductor. A representative example of such a laser geometry is the III-V microdisk laser heterogeneously integrated on a silicon waveguide layer, shown in figure 1(b). ${ }^{2}$ This laser geometry allows making ultra-compact light sources, given the high confinement of the optical mode in the gain material, while the coupling to the silicon waveguide layer is achieved through an intra-cavity evanescent coupling scheme. The wavelength of emission is determined by the diameter of the III-V microdisk, which makes it less straightforward to effectively control this emission wavelength. Given these two extreme cases in the design space and their respective advantages and disadvantages, in the remainder of this paper we will present laser geometries that combine the best of both worlds, i.e. high optical confinement in the gain material, combined with a wavelength selective feedback mechanism defined in the silicon waveguide layer. But before that, we will elaborate on the technological challenges to integrate the III-V epitaxial layer structure on top of the silicon waveguide layer.

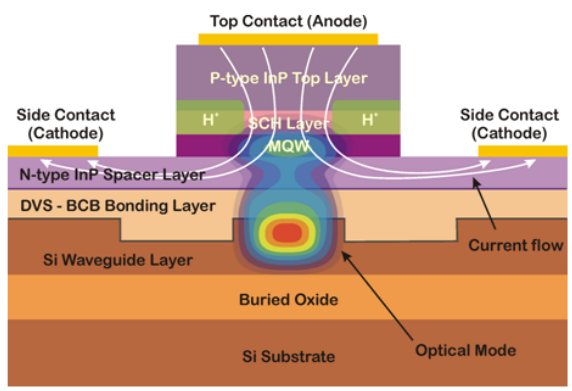

(a)

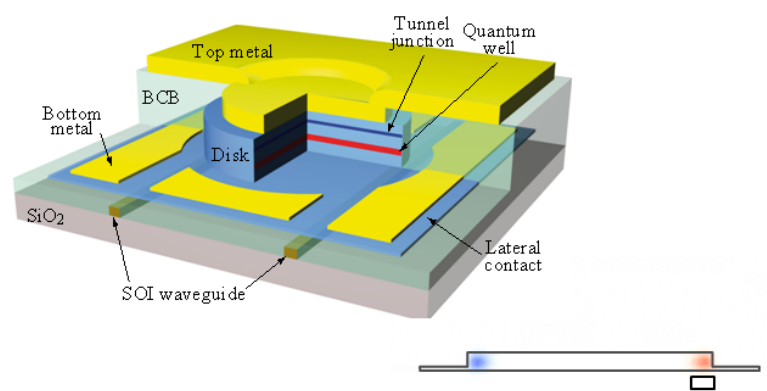

(b)

Figure 1. Two examples of choices made inside the laser design space: a silicon-confined III-V/silicon laser (a) and a III-V confined III-V/silicon laser geometry (b)

\section{HYBRID LASERS INTEGRATION TECHNIQUES}

The integration of III-V material on the SOI platform is a prerequisite for the fabrication of hybrid lasers, but it also presents a technological challenge. In general, the three main approaches for solving this problem are flipchip integration, hetero-epitaxial growth and bonding of III-V material on a SOI wafer. In the case of flip-chip integration, a pre-fabricated photonic component is flipped over and bonded to SOI using solder bumps. This approach has been suggested for the integration of III-V lasers on SOI, ${ }^{3}$ but such integration technique requires precise alignment and sequential attachment of individual devices. It is therefore time-consuming and costly for large scale integration of photonic devices on a single SOI wafer. On the other hand, the hetero-epitaxial growth of III-V on the SOI platform offers a possibility for large scale, high density integration and wafer-scale processing of photonic devices, after the functional III-V layers are grown. This eliminates the need for the precise alignment and allows simultaneous device processing and high-throughput fabrication. However, the lattice mismatch between III-V materials and silicon makes this approach not straightforward, given the formation of defects in the grown epitaxial layers. ${ }^{4}$ Continuous improvements are being made however to mitigate the issues associated with the lattice mismatch, ${ }^{5}$ and it remains to be seen whether this approach is viable in an industrial-scale fabrication process. 
Considering the drawbacks and limitations of flip-chip and hetero-epitaxial growth today, the bonding of III$\mathrm{V}$ material on a SOI wafer remains the most promising and the most commonly used method for the fabrication of hybrid III-V/Si lasers. In this approach, two different integration strategies can be followed. In the first one, a pre-processed, or partially processed photonic component can be bonded on the SOI waveguide platform. Like the flip-chip integration, this approach imposes very stringent alignment requirements. Hybrid lasers based on metallic bonding of pre-fabricated III-V DFB lasers on SOI waveguides were recently demonstrated, ${ }^{6}$ but their fabrication requires a sub-micron precision bonding procedure. Therefore, the preferred strategy is to first bond unprocessed layers of III-V material on the SOI and then perform wafer-scale processing of the devices, by means of standard lithographic procedures. In this way, no precise bonding alignment is required and the simultaneous processing of a multitude of devices on a single SOI wafer is feasible, with a high yield and high integration density.

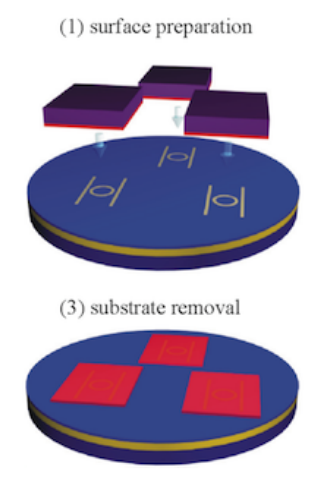

a)

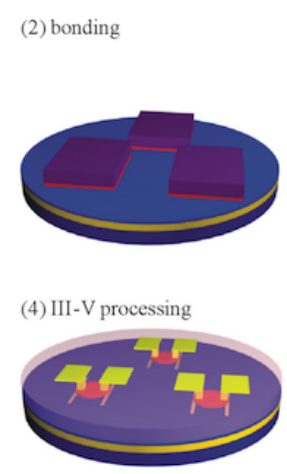

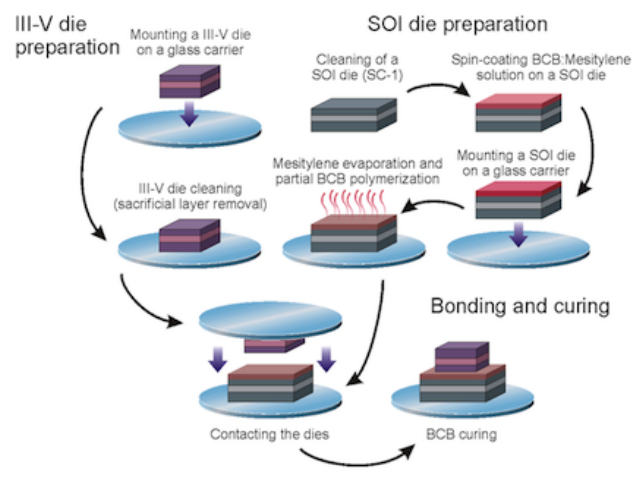

b)

Figure 2. Process flow of the device fabrication with the III-V/SOI die-to-wafer bonding technology (a) and process flow of a die-to-die BCB bonding process (b)

Traditionally, bonding techniques were developed for wafer-to-wafer bonding. However, due to the wafer size mismatch and the fact that most of III-V material is lost in the subsequent processing, this approach is not very cost-effective, unless the largest fraction of the SOI waveguide circuit requires the presence of III-V semiconductor. Alternatively, multiple die-to-wafer bonding gives an opportunity to bond individual III-V dies on specific locations on a SOI wafer where they are actually needed. In this way, III-V material is used in a much more efficient manner. Schematically, this method is illustrated in figure 2(a). Following the surface preparation, individual III-V dies are bonded on a processed SOI wafer with the passive photonic components. After the bonding, the InP substrate is removed by combination of mechanical grinding and chemical wet-etching. An etch stop layer is used to separate the substrate from the functional layers. Subsequently, III-V processing of the opto-electronic components can be done on a wafer-scale.

The two most commonly used bonding techniques are direct (or molecular) bonding and adhesive bonding. Direct bonding is based on intermolecular van der Waals forces that tend to keep molecules together, when they are sufficiently close to each other, usually below $0.5 \mathrm{~nm}$. Therefore, an effective direct bonding between a III-V and SOI wafers requires very flat, ultimately clean surfaces in order to get the different wafers into such an intimate contact. This is a well-known technique, successfully used for fabrication of SOI wafers for almost a decade, with a high yield. The bonding of III-V semiconductors onto processed SOI waveguide wafers can however pose additional problems in terms of yield, given the lower-quality surfaces. Therefore, our research effort focuses on the use of adhesive wafer bonding. Compared to the direct bonding, the use of an adhesive bonding agent generally gives much more relaxed requirements for the bonding surface roughness, topography and particle contamination. Various adhesives can be used for the wafer bonding, ${ }^{7}$ but since it is preferred to have post-bonding processing of III-V material on top of SOI, adhesives that provide sufficiently high thermal budget (up to $400{ }^{\circ} \mathrm{C}$ ) should be used. Therefore, we focus on the use of BCB, given its superior characteristics. ${ }^{8}$ 
BCB is well-known in electronics industry and provides sufficient thermal budget, low optical loss at telecommunication wavelengths and low shrinkage upon curing. Several device demonstrations have been made based on this adhesive bonding approach, including photodetectors ${ }^{9,10}$ and lasers. ${ }^{11,12}$

As a general illustration of a $\mathrm{BCB}$ bonding process, a die-to-die bonding procedure is presented in Figure 2(b). After cleaning the SOI surface (using SC-1 solution), a BCB solution (in mesitylene) is spin-coated on the SOI wafer (or a die). The liquid BCB solution can planarize the SOI wafer topography and sufficiently small particles can be incorporated in the adhesive film, not compromising the bonding quality. After spin-coating, the $\mathrm{BCB}$ is baked at $150{ }^{\circ} \mathrm{C}$ to evaporate the solvent and settle the applied film. After this, a sacrificial layer on top of the III-V die (or wafer) is removed by wet etching and the III-V die is attached on the SOI after which the whole stack is placed in an oven where the $\mathrm{BCB}$ is cured in a nitrogen atmosphere at $250{ }^{\circ} \mathrm{C}$ for 1 hour. Usually, a pressure of $200-300 \mathrm{kPa}$ is applied during the curing.

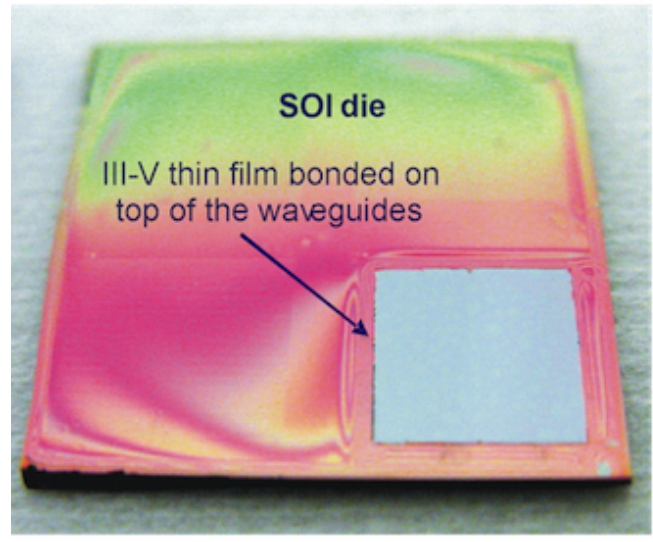

a)

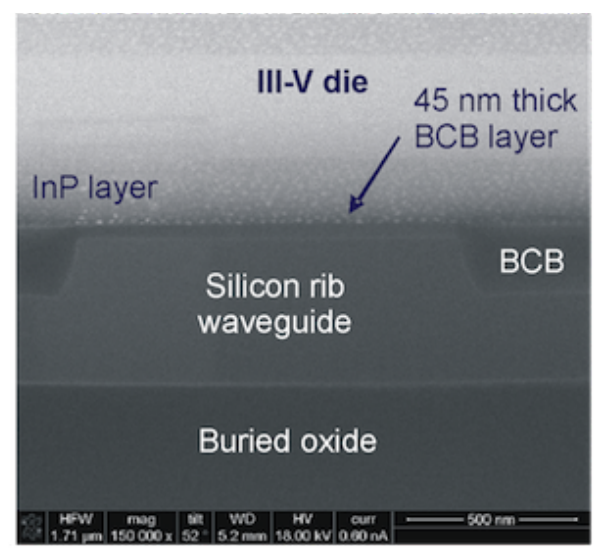

b)

Figure 3. Bonding results in the case of very thin BCB layers: (a) III-V thin film bonded on a patterned SOI die with a very high yield; (b) SEM image of the III-V film on top of the SOI waveguide, with a 45nm-thick BCB bonding layer.

The relaxed wafer quality requirements in the adhesive wafer bonding process are especially valid in the case where the BCB bonding layers are several hundred nanometers thick. However, as the bonding layers become thinner, the requirements become more stringent, as larger particles cannot be tolerated and the SOI wafer topography starts to play a more significant role. Specifically the realization of evanescently-coupled photonic devices requires very thin bonding layers $(<100 \mathrm{~nm})$ with high uniformity. Recently, very thin BCB bonding layers, suitable for the fabrication of evanescently-coupled hybrid III-V/Si lasers have been demonstrated, both in the case of bonding a III-V wafer ${ }^{13}$ and a III-V die ${ }^{14}$ on SOI waveguide circuits.

The uniformity of the bonding layer is of paramount importance. The latest results demonstrate that even in the case of bonding a relatively large III-V die $(8 \mathrm{~mm} \times 7 \mathrm{~mm})$ on top of the SOI waveguides, good BCB layer uniformity can be achieved, retaining high bonding yield and very thin BCB layers (see Figure 3 ). ${ }^{14}$ Variations in the BCB bonding layer thickness are usually within several nanometers and well within the range that is required for evanescently-coupled photonics devices. This shows that the BCB bonding technique is able to match direct bonding when ultra-thin bonding layers are required for evanescently-coupled devices, while also being able to achieve thicker bonding layers for other opto-electronic devices.

\section{COUPLING MECHANISMS}

As mentioned before, an optimal laser cavity design should consist of a section where the optical mode is completely confined to the III-V waveguide layer, while the wavelength selective feedback is provided by structures defined in the silicon. Of course, the laser emission should also be coupled efficiently into the silicon waveguide 
layer. In this section we will present a few laser cavity designs that fulfill these requirements. This includes an adiabatic tapered coupling to transform the optical mode from a III-V confined optical mode to a silicon confined optical mode, a broadband grating based reflector for the realization of a hybrid silicon modelocked laser and the use of resonant grating reflectors for the wavelength selective feedback and outcoupling.

\subsection{Adiabatically tapered coupling}

A relatively straightforward method to achieve a high performance laser cavity is to use an intra-cavity double taper structure, using taper-based mode transformers in both the III-V and silicon waveguides. ${ }^{15}$ Mode coupling occurs in the tapered region, while light generation and amplification take place in the III-V waveguide. This approach allows for the incorporation of wavelength selective feedback structures in the silicon waveguide layer, such as gratings and ring resonators, while it provides at the same time also an efficient way of coupling to a passive silicon waveguide circuit outside the laser cavity. If we consider half of the device structure (figure 4(a)), the structure can be divided into three parts. In the section (1), there is a III-V waveguide that provides optical gain. At right side of it, there is a coupling region that couples light from one waveguide to the other. Section (3) is a silicon waveguide without III-V on top.

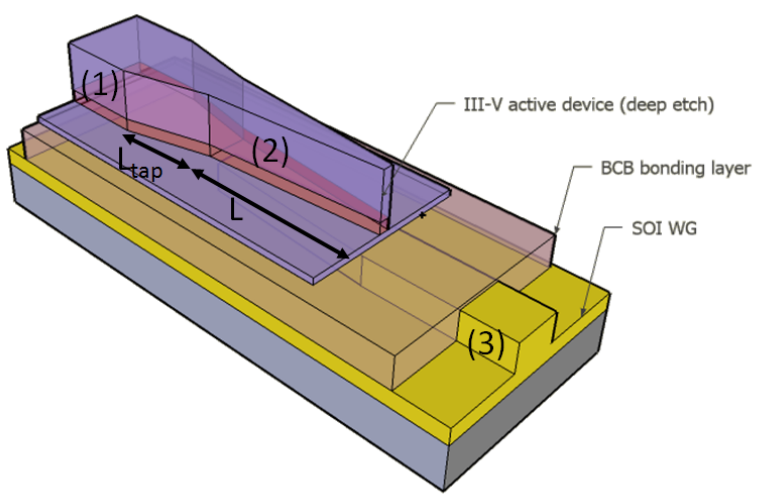

(a)

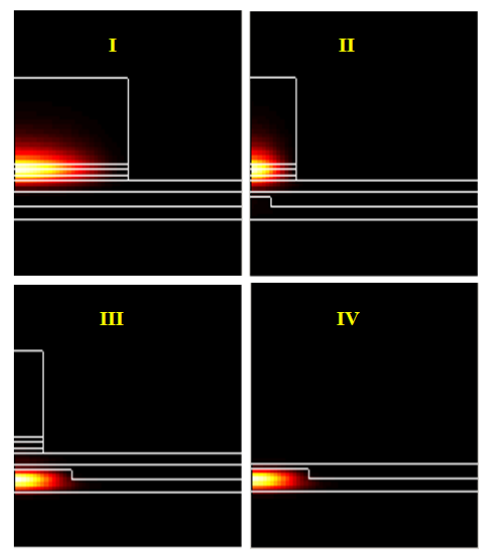

(b)

Figure 4. The schematic of an adiabatically tapered III-V/silicon laser (a) and the evolution of the optical mode profile throughout the taper structure (b)

The III-V region consists of a standard multiple quantum well (QW) double heterostructure, which consists of a p-InGaAs contact layer, a p-InP clad $(1.5 \mu \mathrm{m}), 6$ InGaAsP QW surrounded by two InGaAsP separate confinement heterostructure (SCH) layers (with a total thickness of $320 \mathrm{~nm}$ ), and an n-InP layer (200nm). The SOI substrate consists of a $400 \mathrm{~nm}$ thick silicon waveguide layer on top of a $2 \mu \mathrm{m}$ thick buried oxide layer. The silicon rib waveguides have an etch depth of $180 \mathrm{~nm}$ and a width of $1 \mu \mathrm{m}$. In our design, the underlying silicon rib waveguide (length $L=150 \mu \mathrm{m}$ ) is tapering from $1 \mu \mathrm{m}$ width to $400 \mathrm{~nm}$. The III-V adiabatic taper is a piecewise linear taper tapering from $2 \mu \mathrm{m}$ width downto $900 \mathrm{~nm}$ width (length $L_{t a p}=20 \mu \mathrm{m}$ ) and from $900 \mathrm{~nm}$ width to $500 \mathrm{~nm}$ width again with length $L$. This $500 \mathrm{~nm}$ wide taper tip, combined with the deep etching, makes the fabrication of such a device not straightforward. The required length of the taper depends on the BCB bonding layer thickness. Full vectorial calculations show that, assuming a bonding layer thickness below 100nm and perfect alignment between the silicon waveguide and the III-V waveguide, a taper length $L$ of $150 \mu \mathrm{m}$ should be sufficient for adiabatic mode conversion. A first prototype of such a device, not yet incorporating the wavelength selective feedback structure, was recently demonstrated. ${ }^{15}$ A Fabry-Perot hybrid III-V/silicon laser was realized by defining the mirrors on the passive SOI waveguides by cleaving the SOI wafer. The device was mounted on a temperature controlled stage set to $290 \mathrm{~K}$. The output laser beam is collected by a lens fiber coupled to the silicon waveguide cleaved facet. The coupling losses are estimated to be higher than $5 \mathrm{~dB}$. The L-I curve is shown 
in figure 5a. The laser is electrically pulse-pumped up to $200 \mathrm{~mA}$, with a pulse period and width of $10 \mu$ s and 180ns. The device has a lasing threshold of $45 \mathrm{~mA}$ and a maximum fiber coupled power of $0.75 \mathrm{~mW}$ at both sides. The series resistance is 12 Ohms. The lasing spectrum is shown in figure $5 \mathrm{~b} .{ }^{16}$

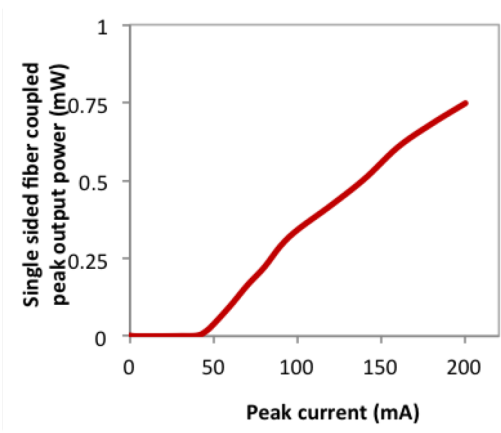

a)

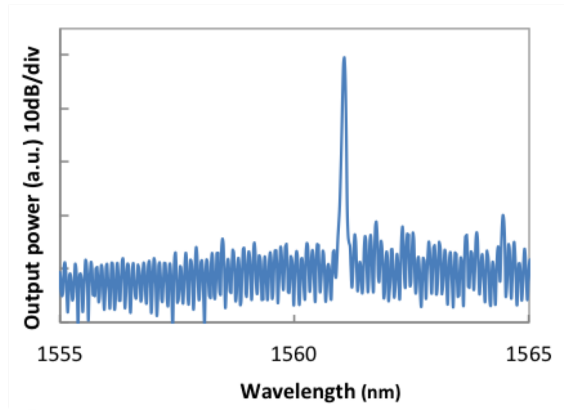

b)

Figure 5. Fiber coupled laser power as a function of drive current (L-I curve) under pulsed operation at 20C (a) and optical spectrum of the laser driven at $55 \mathrm{~mA}$ (b).

\subsection{Broadband coupling for mode-locked lasers}

In contrast with cavities for single longitudinal mode lasers, cavities for mode-locked lasers should support many longitudinal modes to allow for the creation of very short pulses. Therefore, the feedback mechanism in the cavity should be broadband. This relaxes the requirements on the feedback section and allows for more design flexibility, which can be used to create more robust designs in terms of fabrication tolerances. As mentioned in the previous section, the fabrication of taper-based mode converters is not straightforward because of the narrow tips required and the strong dependence of the performance on alignment accuracy and bonding layer thickness. It would thus be beneficial to think of cavities that do not use an adiabatic taper to couple light between the III-V layer and the silicon waveguides. This can be realized in a DBR-based cavity by using the feedback gratings both as reflectors for the cavity mode and counter-directional couplers towards an output silicon wire. This can be achieved using a reflector design such as in figure 6a. In this design, the grating teeth extend from the waveguide layer, which provides for the necessary perturbation to have broadband reflection. The output silicon waveguide however is quite thin $(220 \mathrm{~nm})$ and only barely couples with the III-V wire above it. In this way, one can divert the output light to the SOI circuit without perturbing the lasing mode in the cavity. In figure $6 \mathrm{~b}$ a 3D FDTD simulation result is shown for a 40 period grating (260nm grating period, $50 \%$ fill factor).

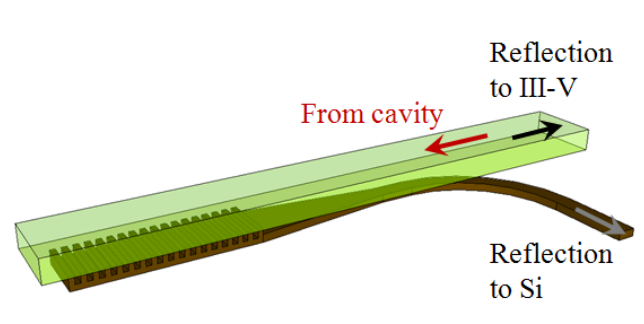

(a)

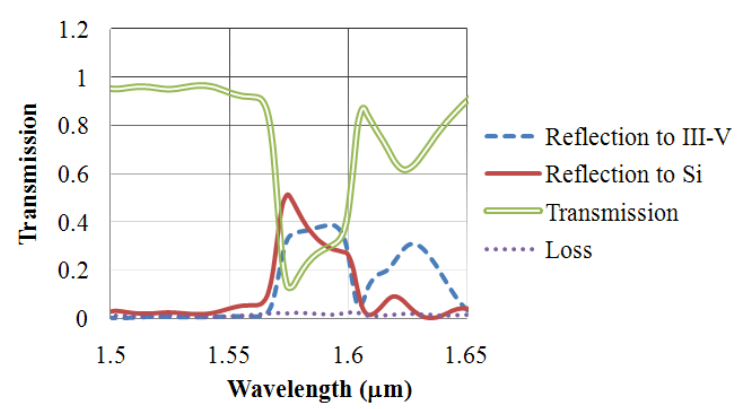

(b)

Figure 6. Layout of the reflector (a) and simulated reflection spectrum of a 40 period grating (b)

\subsection{Resonant mirrors}

To obtain high, narrow-banded reflection using a short grating structure, one can opt for a laser design based on resonant mirrors. ${ }^{17}$ Again, the laser mode inside the gain section is completely confined to a III-V mesa-shaped 
waveguide. At the ends of that gain section, silicon gratings underneath the III-V waveguide will provide optical feedback (fig 7). The silicon grating acts as a periodic perturbation to the III-V waveguide, but because the overlap of the III-V waveguide mode with the grating is weak, this perturbation is typically very small, yielding very long grating reflectors.

To solve this problem, a quarter-wavelength phase shifting section is introduced near the center of the silicon grating, turning it into a resonant cavity at the Bragg wavelength. A limited fraction of light in the III-V waveguide couples to the silicon grating resonator (fig 7-1) and power will build up inside that grating cavity (fig 7-2). Eventually, a significant amount of light will couple back into the III-V waveguide. The light that couples co-directionally to the light incident from the III-V laser cavity will interfere destructively with the latter (fig 7-3) and result in zero overall transmission through the III-V waveguide. The counter-directionally coupling light (fig 7-4) will propagate back into the laser cavity and hence provide optical feedback. Because this reflection mechanism is based on a resonance phenomenon inside the silicon grating, its spectral response will be narrow-banded.

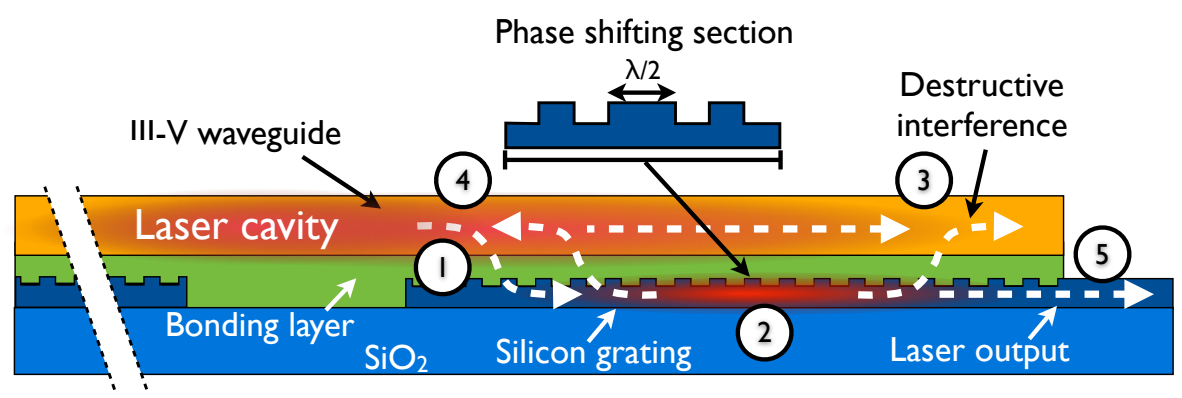

Figure 7 . Side view of hybrid laser with resonant mirrors

The resonant mirror also offers an elegant mechanism to couple the laser-output to a silicon waveguide (fig 7-5). By engineering the position of the quarter-wavelength phase shifting section, one can tune the amount of optical power that leaks from the silicon grating cavity into an output waveguide.

The advantage of this approach is that it doesn't require very thin bonding layers, as the coupling from the III-V waveguide to the silicon grating cavity can be very small, but because the coupling is distributed along the length of the grating, the III-V waveguide mode has to be phase-matched to the resonant grating mode for the reflector to work. This requires careful design of both waveguide geometries.

Figure 8 shows an example of the calculated reflection spectrum for a resonant mirror [blue curve] with only 60 periods (about $20 \mu \mathrm{m}$ long) and a very thick bonding layer $(350 \mathrm{~nm}$ ). The reflection peak is narrow (FWHM is approximately $5 \mathrm{~nm}$ ) with a maximum of more than $93 \%$. The narrow banded reflection spectrum is ideal to select only one desired longitudinal laser mode. As a reference, the red curve shows the reflection spectrum of the exact same mirror but without quarter wavelength phase shifting section. In this case, the maximum reflection is only $3 \%$ at the Bragg Wavelength, suggesting a very weak interaction between the III-V mesa and silicon grating waveguide.

\section{CONCLUSIONS}

The combination of high precision patterning methods in silicon fabs and high gain provided by III-V semiconductors provides a winning combination for advanced lasers. The design space is large and there is ample opportunity for optimization towards specific performance objectives (power, spectral properties, pulsed operation, size, etc.). The critical technological step in hybrid III-V / silicon lasers is obviously the die-to-wafer bonding step. While early approaches suffered from low yield the technology has now become more mature. 


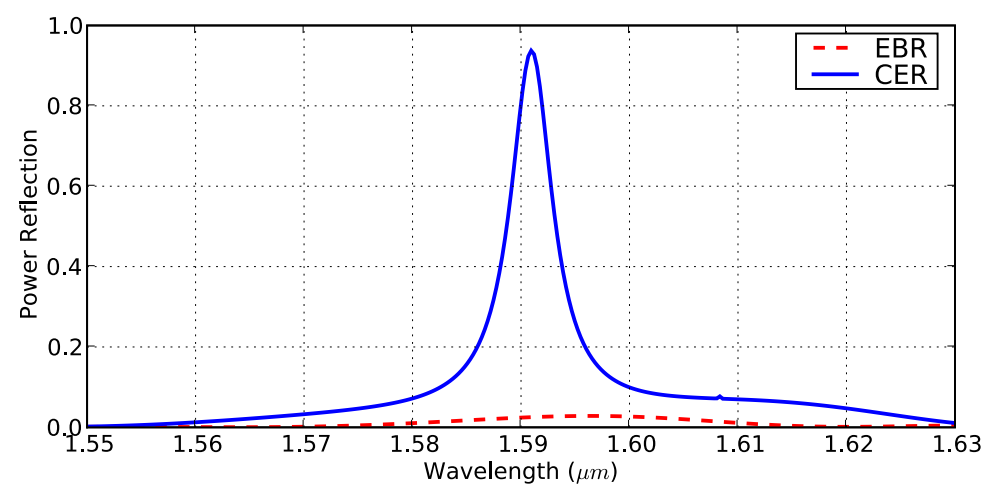

Figure 8. Spectrum of resonant mirror (blue solid curve - Cavity Enhanced Reflector) and associated evanescent Bragg reflector (red dotted curve - EBR)

\section{ACKNOWLEDGEMENTS}

Part of this work was carried out in the framework of the FP7-ICT-HELIOS, the FP7-ICT-HISTORIC project and the national IWT-SBO-Glucosens project. This work was also supported by a grant from Intel Corporation. Y. De Koninck acknowledges the Fund for Scientific Research (FWO-Vlaanderen) for a PhD grant.

\section{REFERENCES}

[1] Fang, A. W., Park, H., Kuo, Y.-h., Jones, R., Cohen, O., Liang, D., Raday, O., Sysak, M. N., Paniccia, M. J., and Bowers, J. E., "Hybrid silicon evanescent devices," MATERIALS TODAY 10(7-8), 28-35 (2007).

[2] Roelkens, G., Liu, L., Liang, D., Jones, R., Fang, A., Koch, B., and Bowers, J., "III-V/silicon photonics for on-chip and inter-chip optical interconnects," LASER \& PHOTONICS REVIEWS 4, 751-779 (Nov. 2010).

[3] Mitze, T., Schnarrenberger, M., Zimmermann, L., Bruns, J., Fidorra, F., Kreissl, J., Janiak, K., Fidorra, S., Heidrich, H., and Petermann, K., "Hybrid integration of III/V lasers on a silicon-on-insulator (SOI) optical board.," in [2005 2nd IEEE International Conference on Group IV Photonics], 210-212 (2005).

[4] Fehly, D., Schlachetzki, A., Bakin, A. S., Guttzeit, A., and Wehmann, H.-H., "Monolithic InGaAsP optoelectronic devices with silicon electronics," IEEE Journal of Quantum Electronics 37, 1246-1252 (2001).

[5] Olsson, F., Xie, M., Lourdudoss, S., Prieto, I., and Postigo, P. A., "Epitaxial lateral overgrowth of InP on Si from nano-openings: Theoretical and experimental indication for defect filtering throughout the grown layer," Journal of Applied Physics 104(9), 093112 (6 pp.) (2008).

[6] Hong, T., Ran, G.-Z., Chen, T., Pan, J.-Q., Chen, W.-X., Wang, Y., Cheng, Y.-B., Liang, S., Zhao, L.-J., Yin, L.-Q., Zhang, J.-H., Wang, W., and Qin, G.-G., "A Selective-Area Metal Bonding InGaAsP-Si Laser," IEEE PHOTONICS TECHNOLOGY LETTERS 22, 1141-1143 (Aug. 2010).

[7] Niklaus, F., Stemme, G., Lu, J. Q., and Gutmann, R. J., "Adhesive wafer bonding," JOURNAL OF APPLIED PHYSICS 99 (Feb. 2006).

[8] Niklaus, F., Andersson, H., Enoksson, P., and Stemme, G., "Low temperature full wafer adhesive bonding of structured wafers," SENSORS AND ACTUATORS A-PHYSICAL 92, 235-241 (Aug. 2001).

[9] Brouckaert, J., Roelkens, G., Van Thourhout, D., and Baets, R., "Compact InAlAs-InGaAs MetalSemiconductorMetal photodetectors integrated on silicon-on-insulator waveguides," IEEE PHOTONICS TECHNOLOGY LETTERS 19(17-20), 1484-1486 (2007).

[10] Sheng, Z., Liu, L., Brouckaert, J., He, S., and Van Thourhout, D., "InGaAs PIN photodetectors integrated on silicon-on-insulator waveguides," OPTICS EXPRESS 18, 1756-1761 (Jan. 2010).

[11] Roelkens, G., Van Thourhout, D., Baets, R., Notzel, R., and Smit, M., "Laser emission and photodetection in an InP/InGaAsP layer integrated on and coupled to a Silicon-on-Insulator waveguide circuit," OPTICS EXPRESS 14, 8154-8159 (Sept. 2006). 
[12] Liu Liu, Spuesens, T., Roelkens, G., Van Thourhout, D., Regreny, P., and Rojo-Romeo, P., "A thermally tunable III-V compound semiconductor microdisk laser integrated on silicon-on-insulator circuits," IEEE Photonics Technology Letters 22(17), 1270-1272 (2010).

[13] Bordel, D., Argoud, M., Augendre, E., Harduin, J., Philippe, P., Olivier, N., Messaoudene, S., Gilbert, K., Grosse, P., Ben Bakir, B., and Fedeli, J., "Direct and Polymer Bonding of III-V to Processed Silicon-OnInsulator for Hybrid Silicon Evanescent Lasers Fabrication," ECS Transactions 33(4), 403-410 (2010).

[14] Stankovic, S., Van Thourhout, D., Roelkens, G., Jones, R., Heck, J., and Sysak, M., "Die-to-Die Adhesive Bonding for Evanescently-Coupled Photonic Devices," ECS Transactions 33(4), 411-420 (2010).

[15] Lamponi, M., Keyvaninia, S., Pommereau, F., Lelarge, F., de Valicourt, G., Roelkens, G., Van Thourhout, D., Bordel, D., Messaoudene, S., Fedeli, J., and Duan, G.-H., "Heterogeneously integrated InP/SOI laser using double tapered single-mode waveguides through InP die to SOI wafer bonding," in [Group IV Photonics], (2010).

[16] Keyvaninia, S., Roelkens, G., and Van Thourhout, D., "Engineering the heterogeneously integrated IIIV/SOI tunable laser," in [14th Annual Symposium of the IEEE Photonics Benelux Chapter], 141-144 (2009).

[17] De Koninck, Y., Roelkens, G., and Baets, R., "Cavity Enhanced Reflector Based Hybrid Silicon Laser," in [IEEE Photonics Society Annual Meeting 2010], (2010). 